\title{
Compensation in Nonsinusoidal, Unbalanced Three-Phase Four-Wire Systems With Active Power-Line Conditioner
}

\author{
P. Salmerón, J. C. Montaño, Senior Member, IEEE, J. R. Vázquez, J. Prieto, and A. Pérez
}

\begin{abstract}
For three-phase four-wire circuits, two compensation criteria have been establishcd: one based on the instantaneous value concept and the other on the average value concept. Thus, according to the instantaneous value concept the non instantaneous power current is reduced, without altering the instantaneous active power. According to the average value concept, the nonactive average-current is reduced, without altering the average power. When the zero-sequence voltage component exists, both compensation types would not enable the zero-sequence (neutral) source current elimination. Then, two approaches are marked in this paper. The first one is for eliminating the non instantaneous power current or the nonactive average-current but the neutral current can still flow. The second one for eliminating the modified non instantaneous power current or the modified nonactive average-current, thus the neutral current component is compensated. According to recent recommendations in this work three-phase systems are considered as four-conductor systems. Experimental results are obtained to confirm the theoretical properties and to show the compensator performance.
\end{abstract}

\section{INTRODUCTION}

$\mathbf{T}$ ODAY, load compensation is considered an important topic in power engineering. Load compensation is the procedure used to obtain the supply currents sinusoidal and balanced or with the same waveform as the respective source voltages (i.e., source currents with low harmonic distortion and unbalance components). Presently, static VAR compensation systems make the former subject like the active power-line conditioners (APLCs) possible. However, there is not a single and universally accepted nonsinusoidal electric power theory to obtain the control algorithm for perfect load compensation.

In general, from the compensation point of view, three-phase systems in distorted and/or unbalanced condition, the analysis of the line current is based on the assumption that any current may be divided into two components: active and reactive. There are two main theories. The first was introduced by Fryze [1]

This work was supported by the "Comision Interministerial de Ciencia y Tecnología (CICYT)" of the Spanish Minister of Education and Culture. It is part of a project covering aspects of power-quality measurements, active power filters, and instantaneous compensation of nonlinear loads.

P. Salmerón, J. R. Vázquez, J. Prieto, and A. Pérez are with the Electrical Engineering Department, University of Huelva, Huelva 21819, Spain (e-mail: patricio@uhu.es).

J. C. Montaño is with IRNAS (CSIC), Sevilla 41080, Spain (e-mail: montano@ irnase.csic.es).

Digital Object Identifier 10.1 109/TPWRD.2004.829150 and is based on the average value concept. The active current components (which are calculated using time-average measurements) are in phase with the source voltages, and the reactive or nonactive current components (which are residual components) are in out of phase with the source voltages. This theory permits to speak of a time-average compensation type to eliminate the nonactive current. The second theory was introduced with the so-called "p-q theory," by Akagi et al. [2], who analyzed the source current based on the instantaneous value concept. 'The value of the instantaneous reactive current is determined at any single time instant, and hence, the corresponding compensating current. This enables instantaneous compensation, using switching devices, which theoretically requires no energy storage elements. Later, many instantaneous power theories emerged thereby that have aided to make up the state of the art on electric power [3]-[13].

A problem was detected when the source voltages included a zero-phase sequence component. In both compensation modes, the neutral-wire source-current elimination is not always possible. In fact, when the zero-phase voltage source exists, neither of these compensation types can guarantee the elimination of the neutral current in three-phase four-wire systems. Besides, the analysis of three-phase systems is treated as a special type of a four-conductor system where the line voltage is characterized by the voltages from the conductors to the virtual start point. Thus, the line voltages and the line currents will be represented as a four-entries vectors. The above definition leads to the simplest set of equations for describing the system behavior.

Two approaches are marked in this paper. The first one is for eliminating the noninstantaneous power current [time-instantaneous compensation (TIC)] or eliminating nonactive current component [time-average compensation (TAC)]; thus, neutral current can still flow. The second one proposes to eliminate the modified noninstantaneous power current (mTIC) or the modified nonactive current (mTAC), then the neutral current components is compensated. A practical case has been developed to confirm the theoretical properties and show the performance of control strategies to an active compensator.

In Section II, the definition of electrical quantities commonly used in nonsinusoidal three-phase systems is introduced. Section III relates the principle of TIC and compensating currents for the above approaches that are obtained. Section IV treats the same items of Section III but applied to the principle of TAC. Section V presents the principal results of an experimental prototype designed for testing these concepts. Finally, Section VI presents some conclusions. 


\section{PRELIMINARIES}

In general, a polyphase system can be treated as a special type of a multiconductor system with particular symmetry properties [3]. In fact, in this paper, a three-phase four-wire system will be treated as a four-conductor system. The line current is the current in the conductors of the system, and the vector of line currents $i_{L 1}, i_{L 2}, i_{L 3}, i_{L 4}$ can be represented as

$$
\boldsymbol{i}=\left[\begin{array}{llll}
i_{L 1} & i_{L 2} & i_{L 3} & i_{L 4}
\end{array}\right]^{T} ; \quad \mathbf{1} \cdot \boldsymbol{i}=\left[\begin{array}{llll}
1 & 1 & 1 & 1
\end{array}\right]\left[\begin{array}{c}
i_{L 1} \\
i_{L 2} \\
i_{L 3} \\
i_{L 4}
\end{array}\right]=0 .
$$

Because of the Kirchhoff current law, some of these components are a linear combination of the others, since the addition of the currents is zero. Mathematically, only (4-1) components are sufficient to describe the current vector. However, in three-phase four-wire circuits, the use of the four components often leads to a more straightforward and convenient formulation of the equations describing the system behavior. In the same way, with regard to the voltage, it is convenient and interesting to characterize the line voltages by the voltages from the conductors to a virtual star-point (Fig. 1). This virtual star-point is chosen to have the addition of the voltages equal to zero (i.e., with a set of equal impedances in star connection), where the common point is the reference of the voltages. The conductor or line voltages $u_{L 1}, u_{L 2}, u_{L 3}, u_{L 4}$ can be represented as a vector

$$
\boldsymbol{u}=\left[\begin{array}{llll}
u_{L 1} & u_{L 2} & u_{L 3} & u_{L 4}
\end{array}\right]^{T} ; \quad \mathbf{1} \cdot \boldsymbol{u}=0 .
$$

Let $\|\boldsymbol{u}(t)\|=u_{\Sigma}(\mathrm{t}),\|\boldsymbol{i}(t)\|=i_{\Sigma}(\mathrm{t})$ be the norm of the instantaneous vector of line voltages and line currents in a fourconductor system

$$
\begin{aligned}
u_{\Sigma}(t) & =\sqrt{\boldsymbol{u} \cdot \boldsymbol{u}}=\sqrt{\sum_{j=1}^{4} u_{L j}^{2}(t)} ; \quad i_{\Sigma}(t)=\sqrt{\boldsymbol{i} \cdot \boldsymbol{i}} \\
& =\sqrt{\sum_{j=1}^{4} i_{L j}^{2}(t) .}
\end{aligned}
$$

It is habitual, the name "collective" instantaneous norm and the associated subscript " $\Sigma$ " in these magnitudes in order to distinguish the global values in four-conductor systems. Thus, the collective instantaneous power at a cross section of a fourconductor system as the dot product of the line voltages vector and line currents vector at that section

$$
p_{\Sigma}(t)=\boldsymbol{u} \cdot \boldsymbol{i} .
$$

The average value concept for three-phase systems, in contrast to that of the instantaneous value, has been based on timeaveraged quantities developed in both the frequency-domain and the time-domain. Under stationary and periodic conditions, the average value of the instantaneous active power and the root mean square (rms) value of voltage and current vectors, corresponding to the fundamental period $T$, are usually defined. In fact, under periodic conditions, for an observation interval $\mathrm{T}$

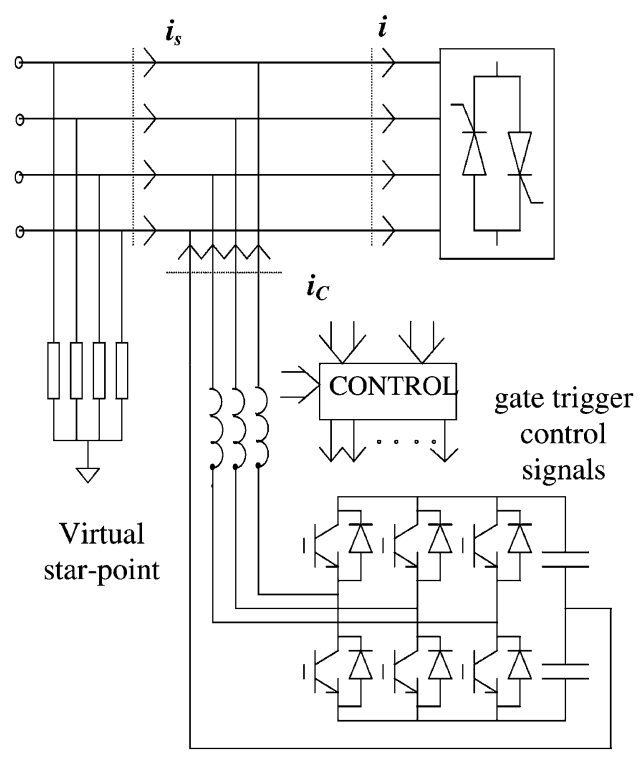

Fig. 1. Three-phase four-wire power system with a shunt active power-line conditioner as compensator.

which coincides with the period or an integer multiple of the period, the mean square value or rms value over the time interval $\mathrm{T}$ is given by

$$
\begin{aligned}
U_{\Sigma} & =\sqrt{\overline{\|\boldsymbol{u}(t)\|^{2}}}=\sqrt{\frac{1}{T} \int_{t-T}^{t}\left(\sum_{j=1}^{4} u_{L j}^{2}(\tau)\right) d \tau} \\
& =\sqrt{\sum_{j=1}^{4} U_{L j}^{2}}
\end{aligned}
$$

for the voltage vector (2) and

$$
I_{\Sigma}=\sqrt{\overline{\|\boldsymbol{i}(\boldsymbol{t})\|^{2}}}=\sqrt{\frac{1}{T} \int_{t-T}^{t}\left(\sum_{j=1}^{4} i_{L j}^{2}(\tau)\right) d \tau}=\sqrt{\sum_{j=1}^{4} I_{L j}^{2}}
$$

for the current vector (1).

The instantaneous power (4) can be averaged over a observation interval $\mathrm{T}$ equal to the period under periodic conditions. In this case, the quantity is the active power

$$
P_{\Sigma}=\frac{1}{T} \int_{T} p_{\Sigma}(\tau) d \tau
$$

Fig. 1 shows the power system with source voltages, generic three-phase load, the virtual star-point for voltage reference, and the static compensator [an active power-line conditioner (APLC)]. The voltage source and load and compensation currents are described in vector form.

A convenient control strategy permits the APLC to inject the compensating currents that improve the source energy efficiency; two compensation methods and two approaches are possible as will be seen in short. 


\section{Time-InStANTANEOUS COMPENSATION}

For a given source voltage vector $\boldsymbol{u}(t)$, there are infinity current vectors $\boldsymbol{i}(t)$, corresponding to the incoming instantaneous power $p_{\Sigma}(t)$ at time $t$. The instantaneous power theory treats on the optimal current vector which delivers the instantaneous power $p_{\Sigma}(t)$ [4]. Here, optimal is employed in the sense of minimum instantaneous norm. This planning yields a method of compensation: time-instantaneous compensation (TIC). When the load is time-variant and the transient state is prevalent, this type of compensation mode is especially appropriated.

\section{A. First Approach}

According to the first approach, for a given source voltage $\boldsymbol{u}(\boldsymbol{t})$, the objective consists in obtaining the current vector $\boldsymbol{i}(\boldsymbol{t})$ with minimum instantaneous norm that delivers the incoming instantaneous power to the load and satisfies the current Kirchhoff's law. The problem is solved by means of the Lagrange multiplier technique. Namely

- Minimize

$$
i \cdot i \text {. }
$$

- Subject to

$$
p_{\Sigma}(t)=\boldsymbol{u} \cdot \boldsymbol{i}
$$

and verifying the Kirchhoff's current law

$$
\mathbf{1} \cdot \boldsymbol{i}=\mathbf{0} ; \quad \mathbf{1}=\left[\begin{array}{llll}
1 & 1 & 1 & 1
\end{array}\right]^{T} .
$$

- The solution of the Lagrange's method gives

$$
\boldsymbol{i}_{\boldsymbol{p}}=\frac{p_{\Sigma}(t)}{\|\boldsymbol{u}(t)\|^{2}} \boldsymbol{u}(t)
$$

The current vector defined by (11), $\boldsymbol{i}_{\boldsymbol{p}}$, is named the vector of the instantaneous power currents which is considered today the technically correct terminology and constitutes the basis of the instantaneous power theory. In general, line current waveforms of $\boldsymbol{i}_{p}(t)$ are different to the corresponding line voltages waveforms of $\boldsymbol{u}(\boldsymbol{t})$. According to the first approach, maximum time-instantaneous compensation is obtained using an APLC that generates the compensating current vector

$$
\boldsymbol{i}_{\boldsymbol{c}}=\boldsymbol{i}-\boldsymbol{i}_{p}
$$

thus, after compensation, the source current $\boldsymbol{i}_{\boldsymbol{s}}$ coincides with the instantaneous power current $\boldsymbol{i}_{p}(t)$

$$
\boldsymbol{i}_{\boldsymbol{s}} \equiv \boldsymbol{i}_{p}=\frac{p_{\Sigma}}{u_{\Sigma}^{2}}=\boldsymbol{u}
$$

The instantaneous power generated by the compensator is zero, that is, according to (11)

$$
p_{c \Sigma}=\boldsymbol{u} \cdot \boldsymbol{i}_{\boldsymbol{c}}=\boldsymbol{u} \cdot \boldsymbol{i}-\frac{p_{\Sigma}}{u_{\Sigma}^{2}} \boldsymbol{u} \cdot \boldsymbol{u}=0 .
$$

In this compensation process, the instantaneous power delivered within the compensator is zero $p_{c \Sigma}(t)=0$. It is considered a compensation method, which does not use energy storage elements.

Although the instantaneous norm of the supply current can be minimized without affecting the instantaneous power, when the TIC is applied, the zero-sequence current component still exists, and it is proportional to the zero-sequence voltage. In fact, the neutral supply current, after compensation is

$$
\boldsymbol{i}_{s 4}=\frac{p_{\Sigma}}{u_{\Sigma}^{2}} u_{L 4} \neq 0
$$

which means that a no null neutral line current can still exist after compensation.

\section{B. Second Approach}

According to the second approach, for a given source voltage, the objective is to obtain the current vector $\boldsymbol{i}_{p}(t)$ with minimum instantaneous norm that delivers to the load the incoming instantaneous power with zero neutral current. In this case, a new condition is included in the optimization process (8)-(9), that is

$$
\mathbf{1}_{4} \cdot \boldsymbol{i}=0 ; \quad \mathbf{1}_{4}=\left[\begin{array}{llll}
0 & 0 & 0 & 1
\end{array}\right]^{T} .
$$

Now, conditions (9), (10), and (16) restrict the minimization of objective function (8). The solution obtained by the Lagrange's method gives

$$
\boldsymbol{i}_{p v}=\frac{p_{\Sigma}}{v_{\Sigma}^{2}} \boldsymbol{v}
$$

where $\boldsymbol{v}$ is defined as the voltage vector without zero sequencephase components

$$
\boldsymbol{v}=\left[u_{L 1}+\frac{u_{L 4}}{3} u_{L 2}+\frac{u_{L 4}}{3} u_{L 3}+\frac{u_{L 4}}{3} u_{L 4}-u_{L 4}\right]^{T}
$$

with instantaneous norm

$$
v_{\Sigma}=\sqrt{u_{\Sigma}^{2}-\frac{4}{3} u_{L 4}^{2}} .
$$

Current vector (17) is the modified instantaneous power current. The APLC, in a mTIC mode within the second approach, must inject the compensating current

$$
\boldsymbol{i}_{c}=\boldsymbol{i}-\boldsymbol{i}_{p v} .
$$

Then, the source current is given by

$$
\boldsymbol{i}_{s}=i_{p v}=\frac{p_{\Sigma}}{v_{\Sigma}^{2}} \boldsymbol{v}
$$

with null neutral current

$$
i_{s L 4}=\frac{p_{\Sigma}}{v_{\Sigma}^{2}} v_{L 4}=0 .
$$

The instantaneous source current vector $\boldsymbol{i}_{\boldsymbol{s} v}$ transfers the collective instantaneous power $p_{\Sigma}(t)$ and the instantaneous power delivered by the compensator is zero, that is, according to (20) and (21)

$$
p_{c \Sigma}=\boldsymbol{u} \cdot \boldsymbol{i}_{c}=\boldsymbol{u} \cdot\left(\boldsymbol{i}-\frac{p_{\Sigma}}{v_{\Sigma}^{2}} \boldsymbol{v}\right)=0
$$

since it fulfills (24)

$$
\begin{aligned}
\boldsymbol{u} & =\boldsymbol{v}+\boldsymbol{v}_{\boldsymbol{z}} ; \quad u_{\Sigma}^{2}=v_{\Sigma}^{2}+v_{z \Sigma}^{2} \\
\boldsymbol{v}_{\boldsymbol{z}} & =\left[\begin{array}{lll}
\frac{-u_{L 4}}{3} & \frac{-u_{L 4}}{3} & \frac{-u_{L 4}}{3} u_{L 4}
\end{array}\right]^{T} .
\end{aligned}
$$

The supply source current after instantaneous compensation is free of zero-sequence current; thus, after compensation, the 
neutral current is eliminated in coincidence with the objectives and criteria of the instantaneous power theories [5]-[13].

\section{TIME-Average COMPENSATION}

The principal objective of TAC, is the optimization of the average power transfer from source to load [i.e., the source current must deliver to the external circuit (compensator and load) only the average power]. As in Section III, two approaches are considered.

\section{A. First Approach}

According to the first approach, the objective is to obtain the current vector $\boldsymbol{i}_{\boldsymbol{a}}(t)$ with minimum rms value that transfers to the load the incoming average power for a given source voltage. The problem is solved by means of the Lagrange multiplier technique.

- Minimum condition (objective function)

$$
\frac{1}{T} \int_{T} \boldsymbol{i} \cdot \boldsymbol{i} d t
$$

- Satisfying condition

$$
\frac{1}{T} \int_{T} \boldsymbol{u} \cdot \boldsymbol{i} d t=P_{\Sigma}
$$

- Verifying the Kirchhoff's current law

$$
\mathbf{1} \cdot \boldsymbol{i}=\mathbf{0} ; \quad \mathbf{1}=\left[\begin{array}{llll}
1 & 1 & 1 & 1
\end{array}\right]^{T} .
$$

The solution of the Lagrange's method gives

$$
\boldsymbol{i}_{a}=G \cdot \boldsymbol{u}
$$

which defines those line currents $\boldsymbol{i}_{a}$ that would be absorbed by an equivalent symmetric resistive load having the same average power consumption as the actual load at voltage $\boldsymbol{u}$. So, the load presents an equivalent conductance defined by

$$
G=\frac{P_{\Sigma}}{U_{\Sigma}^{2}}
$$

The compensating current

$$
\boldsymbol{i}_{c}=\boldsymbol{i}-\boldsymbol{i}_{a}
$$

constitutes the current vector supplied by the APLC, and the source current after compensation is

$$
\boldsymbol{i}_{S}=G \cdot \boldsymbol{u}
$$

Moreover

$$
p_{c \Sigma}(t)=\boldsymbol{u} \cdot \boldsymbol{i}_{c}=p_{\Sigma}(t)-\frac{P_{\Sigma}}{U_{\Sigma}^{2}} u_{\Sigma}^{2}
$$

TABLE I

DifFERENT COMPENSATION TyPES IN THREE-PHASE, Four-Wire Systems

\begin{tabular}{c|c|c}
\hline $\begin{array}{c}\text { COMPENSATION } \\
\text { TYPES }\end{array}$ & $\begin{array}{c}\text { FIRST } \\
\text { APPR. } \\
i_{S 4} \neq 0\end{array}$ & $\begin{array}{c}\text { SECOND } \\
\text { APPR. } \\
i_{S 4}=0\end{array}$ \\
\hline $\begin{array}{c}\text { TIC } \\
\text { Compensation without } \\
\text { energy storage, } p_{c \Sigma}(t)=0\end{array}$ & $\boldsymbol{i}_{C}=\boldsymbol{i}-\boldsymbol{i}_{p}$ & $\boldsymbol{i}_{C}=\boldsymbol{i}-\boldsymbol{i}_{p v}$ \\
\hline $\begin{array}{c}\text { TAC } \\
\text { Compensation with }\end{array}$ & $\boldsymbol{p}_{\Sigma}$ & $\boldsymbol{i}_{\Sigma}^{2} \boldsymbol{u}$ \\
$\begin{array}{c}\boldsymbol{i}_{S}=\frac{p_{\Sigma}}{v_{\Sigma}^{2}} \boldsymbol{v} \\
\text { energy storage, } p_{c \Sigma}(t) \neq 0, \boldsymbol{i}_{a} \\
<p_{c \Sigma}(t)>=0\end{array}$ & $\boldsymbol{i}_{S}=\frac{P_{\Sigma}}{U_{\Sigma}^{2}} \boldsymbol{u}$ & $\boldsymbol{i}_{C}=\boldsymbol{i}-\boldsymbol{i}_{a v}$ \\
\hline
\end{tabular}

is the instantaneous active power flowing through the compensator, according to (27) and (29), which has an average value equal to zero

$$
\frac{1}{T} \int_{T} p_{c \Sigma}(t) d t=0
$$

However, although no power dissipation exists in the compensator, the source side neutral current is not perfectly controlled to zero, thus according (30)

$$
{ }^{\boldsymbol{i}_{4}}=G u_{L 4} \neq 0 \text {. }
$$

\section{B. Second Approach}

As a second approach, for a given source voltage, the objective is to obtain a current vector $\boldsymbol{i}_{\boldsymbol{a} v}(t)$ (modified active current) with minimum rms value that delivers the incoming average power to the load with zero neutral-wire current. In this case, the optimization process (25)-(26), must include a new condition

$$
\mathbf{1}_{4} \cdot \boldsymbol{i}=0
$$

The solution of Lagrange's method gives for the source current after compensation

$$
i_{\boldsymbol{s} v}=G_{v} \boldsymbol{v}
$$

where

$$
G_{v}=\frac{P_{\Sigma}}{V_{\Sigma}^{2}}
$$

is the modified equivalent conductance of the load.

In this case, the line currents can be controlled for null zero-sequence component and the instantaneous power flowing through the compensator

$$
p_{c \Sigma}=\boldsymbol{u} \cdot \boldsymbol{i}_{c}=p_{\Sigma}-\frac{P_{\Sigma}}{V_{\Sigma}^{2}} v^{2}
$$

and also presents an average value equal to zero. 


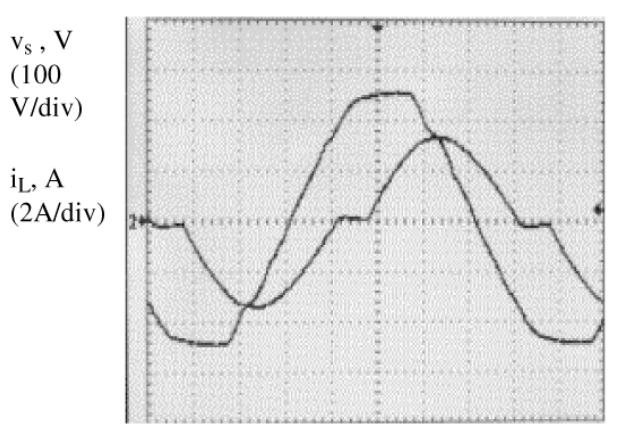

a) Time $(2.5 \mathrm{~ms} / \mathrm{div})$

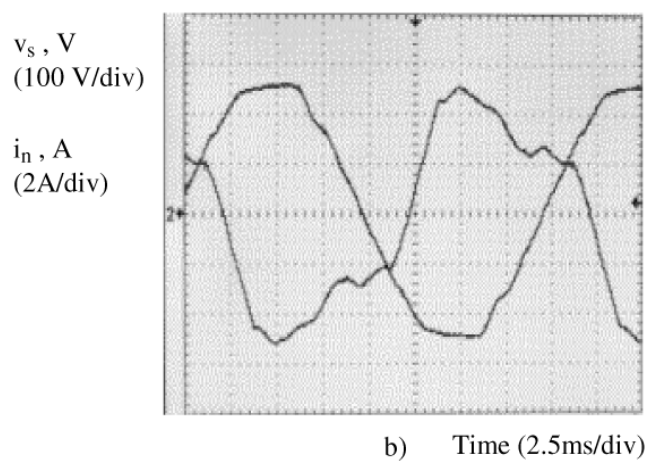

Fig. 2. (a) Source voltage and load current of phase 1. (b) Source voltage and neutral-wire current of practical case.

Table I summarizes the two approaches proposed for both compensation types.

\section{EXPERIMENTAL RESULTS}

The above study was applied to an experimental platform, which includes a real-time controlled APLC and different nonlinear three-phase loads. A digital signal processor (DSP) controller board to carry out the different control strategies of compensation was used. A nonlinear load in star connection with neutral wire of $1 \mathrm{~kW}$ is supplied by the main. Each load-leg contains bidirectional thyristors and series resistors and inductors with different values to obtain an asymmetrical load (Fig. 1). The power circuit of APLC is built from a voltage source inverter with Semikron's insulated gate bipolar transistor (IGBT) modules, model SKM50GB123. Appropriate Hall sensors capture load voltage, load current, and compensator current signals, necessities for the control system. The relationships of Figs. 2-6 show experimental results obtained in the power laboratory, which are visualized in a digital oscilloscope. Fig. 2(a) shows phase 1 waveforms of voltage and load current and Fig. 2(b) shows the neutral-wire current. In the experiment, a high neutral-wire current exists due to load unbalance and voltage asymmetry of the main supply. Fig. 3 shows resulting waveforms of the instantaneous compensation process corresponding to studied approaches. Fig. 3(a) shows the source current of phase 1 for instantaneous compensation without cancelling the full zero-phase current component (first approach). The source neutral-wire current is shown in Fig. 3(b). For the first approach, a neutral current still exists after compensation. Fig. 4(a) and (b) show waveforms resulting for the second approach. When the compensator is connected, the source zero-phase current is zero for any change of the

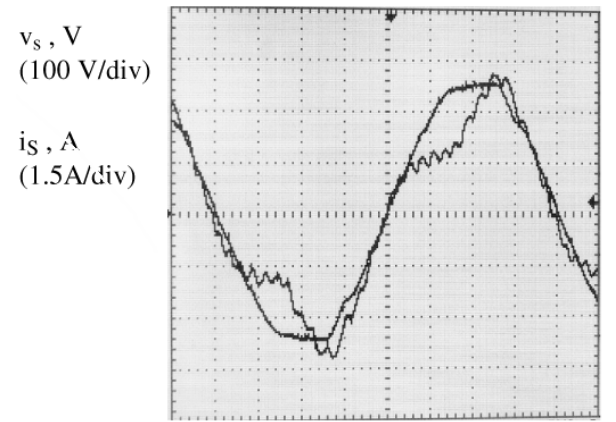

a) Time $(2.5 \mathrm{~ms} / \mathrm{div})$

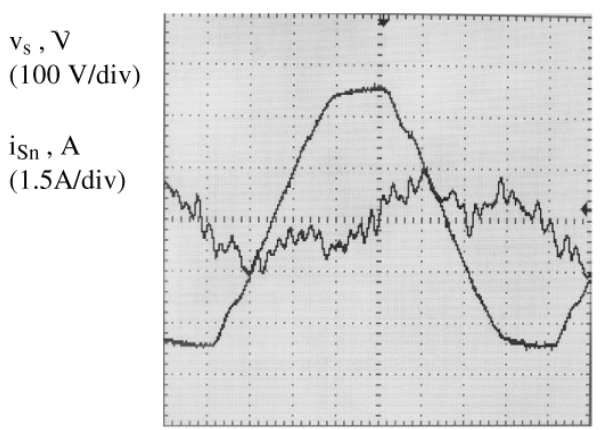

b) Time $(2.5 \mathrm{~ms} / \mathrm{div})$

Fig. 3. Waveforms of time-instantaneous compensation from first approach. (a) source current of phase 1. (b) Source neutral-wire current.

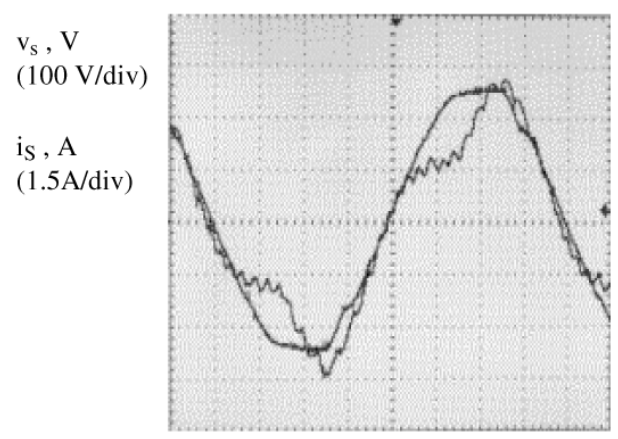

a) Time $(2.5 \mathrm{~ms} / \mathrm{div})$

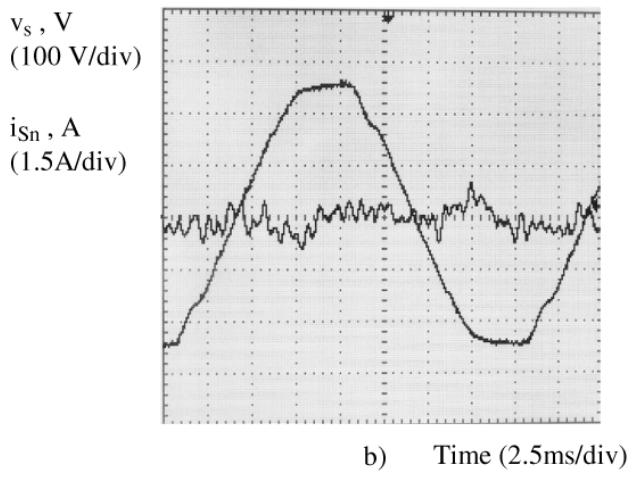

Fig. 4. Waveforms of time-instantaneous compensation from second approach. (a) Source current of phase 1. (b) Source neutral-wire current.

load. The erratic amplitude of the neutral current is due to the threshold band imposed by the PWM control.

Fig. 5 shows the resulting waveforms for the TAC process at two approaches. Fig. 5(a) presents phase 1 source current of time-average compensation without cancellation of zero-phase 


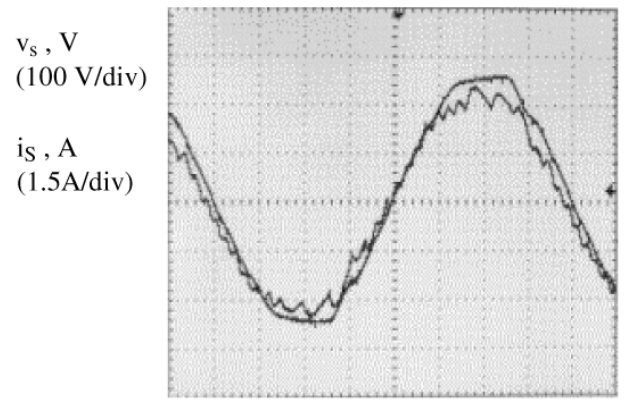

a) Time $(2.5 \mathrm{~ms} / \mathrm{div})$

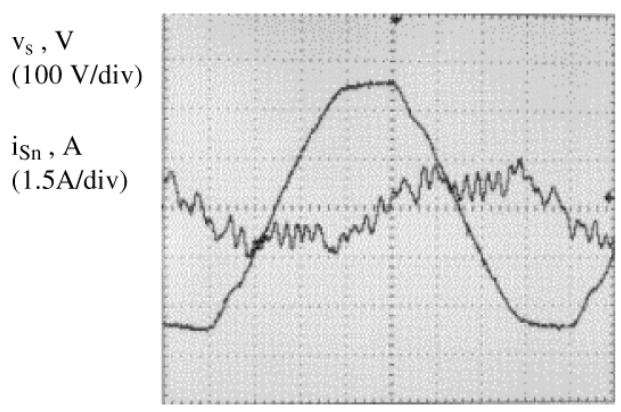

b) Time $(2.5 \mathrm{~ms} / \mathrm{div})$

Fig. 5. Waveforms of time-average compensation from first approach. (a) Source current of phase 1. (b) Source neutral-wire current.

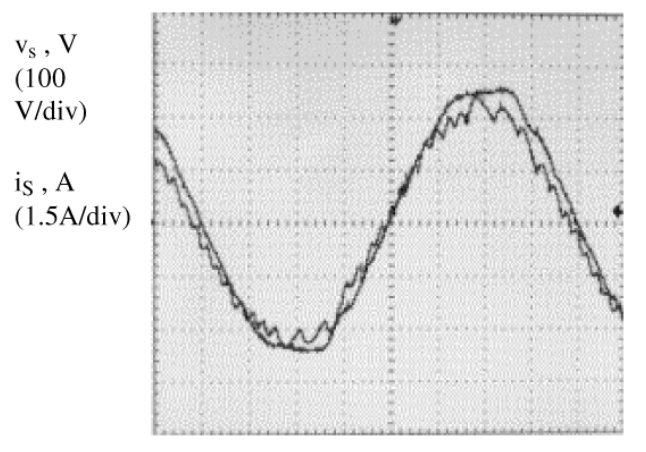

a) Time $(2.5 \mathrm{~ms} / \mathrm{div})$

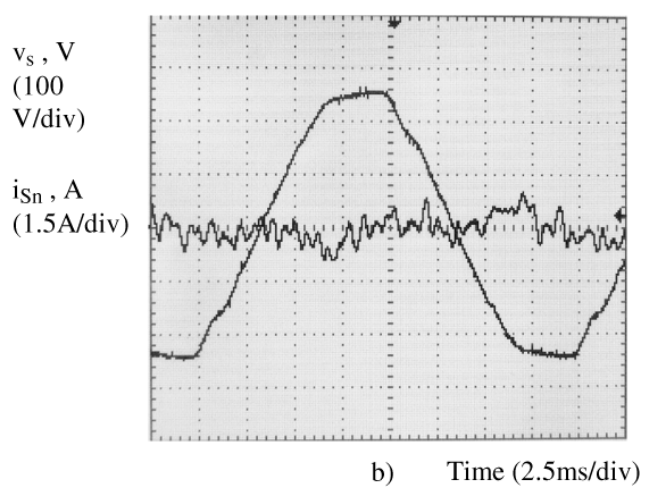

Fig. 6. Waveforms of time-average compensation from second approach. (a) Source current of phase 1. (b) Source neutral-wire current.

current component (first approach). Fig. 5(b) shows neutral current for the first approach; there never is zero. Corresponding to the second approach, Fig. 6(a) shows phase 1 of the source current and Fig. 6(b) shows the neutral current on the source side with the zero-phase current component cancelled.

\section{CONCLUSION}

Analysis of the line current vector, according to the well-established instantaneous and time-averaged power concepts for three-phase systems, is reviewed in this paper. The present discussion is focused on three-phase four-wire load compensation when the zero-sequence voltage component exists. Two compensation criteria are discussed: one based on the instantaneous value concept where the noninstantaneous power current is eliminated and the other on the average value concept where the nonactive current is eliminated. When the zero-phase voltage source exists, neither of these compensation types can guarantee the elimination of the neutral current in three-phase four-wire systems. Two approaches are marked in this paper. The first one is for eliminating directly the noninstantaneous power current (TIC) or eliminating nonactive current component (TAC); thus neutral current can still flow. The second one proposes to eliminate the noninstantaneous power current modified (mTIC) or nonactive current modified (mTAC), then the neutral current components are compensated. Thus, a new compensating current in each compensation mode is introduced, coordinating the points of view expressed in [7], [9], [11], and [13]. According to recent recommendations in this work, three-phase systems are considered as four-conductor systems.

Finally, experimental results of a practical case for checking those approaches are presented.

\section{REFERENCES}

[1] S. Fryze, "Active, reactive and apparent powers in circuits with distorted voltages and currents," ETZ, vol. 53, pp. 596-599, 1932.

[2] H. Akagi, Y. Kanazawa, and A. Nabae, "Instantaneous reactive power compensators comprising switching devices without energy storage components," IEEE Trans. Ind. Applicat., vol. IA-20, pp. 625-630, May/June 1984.

[3] M. Depenbrock, "Quantities of a multiterminal circuit determined on the basis of Kirchhoff's laws," in Proc. 4th Int. Workshop Power Definitions Measurements Under Non-Sinusoidal Conditions, Italy, 1997, pp. 29-35.

[4] J. L. Willems, "Critical analysis of the concepts of instantaneous power current and of active current," in Proc. Europe. Trans. Elect. Power, vol. 8, July/Aug. 1998.

[5] L. Rossetto and P. Tenti, "Evaluation of instantaneous power terms in multi-phase systems: Techniques and application to power-conditioning equipment," in Proc. Eur. Trans. Elect. Power, vol. 4, Nov./Dec. 1994, pp. $469-475$.

[6] A. Nabae and T. Tanaka, "A new definition of instantaneous active-reactive current and power based on instantaneous space vectors on polar coordinates in three-phase circuits," IEEE Trans. Power Delivery, vol. 11, pp. 1238-43, July 1996.

[7] A. Nabae, H. Nakano, and S. Togasawa, "An instantaneous distortion current compensator without any coordinate transformation," in Proc. IEEJ Int. Power Electron. Conf., Yokohama, Japan, 1995, pp. 1651-1655.

[8] F. Z. Peng and J.-S. Lai, "Generalized instantaneous reactive power theory for three-phase power systems," IEEE Trans. Instrum. Meas., vol. 45, no. 1, pp. 293-297, Feb. 1996.

[9] J. C. Montaño and P. Salmerón, "Instantaneous and full compensation in three-phase systems," IEEE Trans. Power Delivery, vol. 13, pp. 1342-47, Oct. 1998.

[10] —, "Identification of instantaneous current components in three-phase systems," in Proc. Inst. Elect. Eng., Sci., Sept. 1999, pp. 227-233.

[11] H. Akagi, S. Ogasawara, and H. Kim, "The theory of instantaneous power in three-phase four-wire systems: A comprehensive approach," in Proc. Conf. Rec. IEEE Ind. Applicat. Conf., vol. 1, 1999, pp. 431-439.

[12] H. Kim, F. Blaabgerg, B. Bak-Jensen, and J. Choi. Novel instantaneous power compensation theory in three-phase systems. presented at Proc. Eur. Conf. Power Electron. Applicat., 2001 